\title{
Tumor miofibroblástico inflamatorio: presentación variable de una misma patología
}

\author{
Inflammatory myofibroblastic tumor: variable presentation of the same pathology
}

Jorge E. Muñoz Moya a , María Olga Alfaro Aguirrea, Mauricio Leiva Silva ${ }^{a}$, Elena Kakarieka Weisskopf ${ }^{b}$, María Teresa López Sáez ${ }^{\text {,d }}$

\author{
aServicio de Cirugía infantil del Hospital Clínico San Borja Arriarán \\ bServicio de Anatomopatología, Hospital Clínico San Borja Arriarán \\ 'Servicio de Urología infantil, Hospital Clínico San Borja Arriarán \\ ${ }^{d}$ Centro de Responsabilidad Quirúrgica infantil, Hospital Clínico San Borja Arriarán
}

Recibido: 21 de septiembre de 2018; Aprobado: 7 de marzo de 2019

\section{Resumen}

El tumor miofibroblástico inflamatorio (TMI) es una neoplasia benigna infrecuente, de comportamiento clínico impredecible. Objetivos: describir 3 casos de TMI diagnosticados entre marzo 2014 y enero 2018 en Hospital Clinico San Borja Arriaran, y realizar una revisión actualizada de la literatura. Caso 1: Adolescente de género masculino de 14 años de edad, hospitalizado por dolor abdominal, diagnosticado de invaginación yeyunoyeyunal secundaria a un tumor de pared intestinal. La histología fue compatible con un tumor miofibroblástico inflamatorio. Caso 2: Adolescente de género femenino, edad 12 años, hospitalizada por neumonía y dolor lumbar en estudio asociado a pérdida de peso. Se diagnosticó una masa retroperitoneal que comprometía el músculo psoas derecho, músculos paravertebrales, vértebras, riñón derecho y diafragma ipsilateral. Se efectuó biopsia por punción cuyo resultado fue compatible con un tumor miofibroblástico inflamatorio. Caso 3: Preadolescente de género femenino de 11 años de edad, hospitalizada para estudio de infección del tracto urinario a repetición. Se identificó un tumor vesical y la biopsia mostró ser compatible con tumor miofibroblástico inflamatorio. Conclusión: Debido al comportamiento variable del tumor miofibroblástico inflamatorio, el manejo de este dependerá de la localización, la expresión del anaplasic like lymphoma (ALK), el comportamiento del tumor y la posibilidad de resección.

\section{Palabras clave:}

Tumor miofibroblástico inflamatorio; marcadores tumorales; tratamiento quirúrgico 


\begin{abstract}
The inflammatory myofibroblastic tumor is an infrequent benign neoplasm with unpredictable clinical behavior. Objectives: to describe three clinical cases at the San Borja Arriarán Clinical Hospital between March 2014 and January 2018 and to carry out an updated review of the literature. Case 1: 14-year-old male adolescent, hospitalized due to abdominal pain, diagnosed with jejunojejunal intussusception secondary to an intestinal wall tumor. The histology was compatible with an inflammatory myofibroblastic tumor. Case 2: 12-year-old female adolescent, hospitalized due to pneumonia and low-back pain under study associated with weight loss. A retroperitoneal mass was diagnosed involving the right psoas muscle, paravertebral muscles, vertebrae, right kidney, and ipsilateral diaphragm. A puncture biopsy was performed and the result was compatible with an inflammatory myofibroblastic tumor. Case 3: 11-year-old female pre-adolescent, hospitalized to study recurrent urinary tract infection. A bladder tumor was identified, and the biopsy showed compatibility with inflammatory myofibroblastic tumor. Conclusion: Due to the variable behavior of the inflammatory myofibroblastic tumor, its management will depend on the location, expression of the anaplastic lymphoma kinase (ALK), tumor behavior, and the resection possibility.
\end{abstract}

\section{Keywords:}

Inflammatory

myofibroblastic tumor;

tumor markers;

surgical treatment

\section{Introducción}

El tumor miofibroblástico inflamatorio (TMI), es un tumor benigno de comportamiento biológico intermedio ${ }^{2,3}$. También es conocido como pseudotumor inflamatorio ${ }^{1}$, granuloma de células plasmáticas, histiocitoma, xantoma, fibroxantoma o fibrosarcoma inflamatorio ${ }^{1,3,5,8}$.

Fue descrito por primera vez en 1937 en un caso con afección pulmonar ${ }^{8,12}$ y desde entonces, se han reportado en distintos sitios anatómicos.

Se presenta con mayor frecuencia en la infancia y en adultos jóvenes ${ }^{2,3}$ y si bien su etiología aún es incierta, se asocia a diversos factores como: traumatismos, infecciones, genética y patologías autoinmunes ${ }^{1,3} . \mathrm{Su}$ diagnóstico definitivo es histológico ${ }^{3,4,5}$ y el tratamiento de elección es la resección quirúrgica ${ }^{1,3,5}$.

Debido a lo infrecuente de esta patología y al diagnóstico de 3 pacientes con este tumor en el Hospital Clínico San Borja Arriarán (HCSBA), el objetivo principal de nuestro trabajo fue describir 3 casos de TMI, diagnosticados entre marzo 2014 y enero 2018. Como objetivo secundario realizamos la revisión y actualización de la literatura acerca de esta patología.

\section{Casos clínicos}

\section{Caso 1}

Adolescente de género masculino, de 14 años de edad, sin antecedentes patológicos.

Consultó en el Servicio de Urgencia Infantil por dolor abdominal tipo cólico de siete días de evolución y vómitos recurrentes las últimas 24 horas. Se evaluó ambulatoriamente donde se dejó con manejo sintomático sin respuesta, por lo que consultó en el HCSBA.
$\mathrm{Al}$ ingreso, el paciente se encontraba en regulares condiciones generales, deshidratado y con mucho dolor. Se inició reanimación con suero fisiológico, analgesia y antieméticos. Destacaron en sus exámenes: leucocitos: $15.240 \mathrm{x} \mathrm{mm}^{3}$; PCR: $1 \mathrm{mg} / \mathrm{dl}$; perfil hepático en rango normal; amilasa: $197 \mathrm{mg} / \mathrm{dl}$; creatinina: 1,52 $\mathrm{mg} / \mathrm{dl}$.

Se realizó Radiografía de abdomen (figura 1A) donde se observó escaso gas distal asociado a imagen en pila de monedas a nivel de mesogastrio y flanco izquierdo (intestino delgado).

Se completó estudio con ecotomografía abdominal donde se observó imagen sugerente de invaginación intestinal yeyunoyeyunal secundaria a pólipo con obstrucción intestinal proximal.

El paciente fue sometido a laparotomía exploradora donde se evidenció invaginación yeyunoyeyunal (figura 1B), asociado a masa de pared intestinal, proyectada hacia el lumen de $5 \times 3,5 \mathrm{~cm}$ de diámetro. Se efectuó resección en bloque (figuras 1C y D).

La histología reveló tumor de células fusadas y ovoides, núcleos elongados con moderado pleomorfismo y algunas células histiocitoides de aspecto ganglionar con algunas mitosis, sin figuras atípicas entremezcladas, con prominente infiltrado inflamatorio linfoplasmocitario y algunos eosinófilos. Pared intestinal con bordes libres. El estudio inmunohistoquímico reveló: Vimentina: positivo; Actina de músculo liso: positivo; Ki-67: positivo 3\%; CD68: positivo en el componente linfoplasmocitario y ALK: positivo débil a moderada en las células tumorales (figura 2).

Los resultados de la lesión fueron compatibles con TMI.

La paciente continúa en control con oncología luego de 8 meses post cirugía, sin episodios de recurrencia. 
Figura 1. A) A nivel de mesogástrio y flanco izquierdo se aprecia asas de intestino delgado con imagen en pila de monedas y escaso gas hacia distal. B) invaginación yeyunoyeyunal secundario a tumor de pared intestinal. C) Tumor visto en su cara endoluminal. D) Corte sagital de tumor que compromete toda la de pared de yeyuno. De aspecto homogéneo y de consistencia gomosa a la palpación.

Figura 2. A y B: tinción de HE. C: actina ML positivo citoplasmático en células fusadas. D: tinción para ALK es positivo débil a moderado en células tumorales.
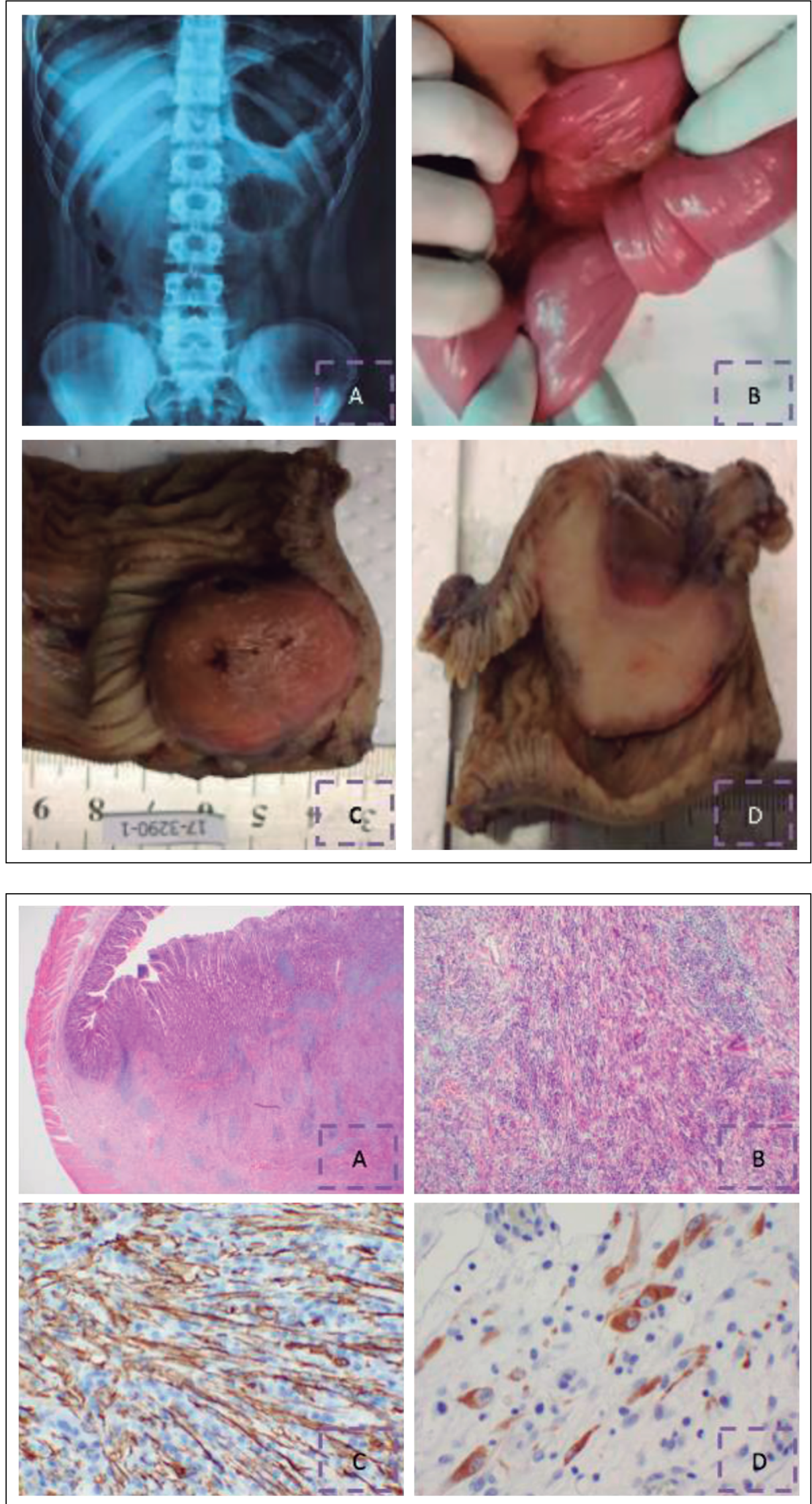


\section{Caso 2}

Adolescente de género femenino, de 12 años de edad, con historia de hemoptisis asociada a pérdida de peso y dolor lumbar de 4 meses de evolución. Se hospitalizó con diagnóstico de neumonía, recibió antibioticoterapia y tuvo una evolución favorable. Debido al dolor lumbar de larga data asociado a pérdida de peso, se realizó tomografía torácica, abdominal y pélvica que reveló tumor retroperitoneal que comprometía psoas derecho, músculos paravertebrales, infiltración ósea en columna vertebral, diafragma derecho y riñón ipsilateral.

Tras realizar biopsia por punción guiada por tomografía, la histología de la muestra mostró escasa a moderada celularidad, con células fusadas de núcleos ovoideos y ondulados y moderado infiltrado constituidos por linfocitos, plasmocitos e histiocitos.

El estudio inmunohistoquímico mostró: Vimentina: positivo; Actina ML: positivo; Desmina: positivo; Miogenina: positivo y ALK: negativo. Los hallazgos fueron compatibles con TMI (figura 3).

Dado su localización y componente infiltrativo, el tumor fue catalogado como irresecable por lo cual se inició terapia con antiinflamatorios: Celecoxib $200 \mathrm{mg}$ cada 12 horas y Prednisona $20 \mathrm{mg}$ cada 12 horas. Si bien, se logró la disminución parcial del tamaño tumoral, la paciente persistió con dolor en región dorsal, por lo que se decidió sumar a la terapia, Infliximab en dosis de $300 \mathrm{mg}$ EV cada 24 horas en esquema de 0-2-6 semanas. La paciente presentó reacción anafiláctica a la administración de la segunda dosis, por lo que, requirió uso de metilprednisolona en el siguiente ciclo. Por recurrencia de efectos adversos, se planteó cambio de Infliximab por otro anticuerpo monoclonal: Adalimumab, en dosis de $40 \mathrm{mg}$ SC cada 15 días, asociado a Celecoxib $200 \mathrm{mg}$ cada 12 horas y Prednisona $20 \mathrm{mg}$ cada 12 horas, lográndose ausencia de la sintomatología y significativa reducción del tamaño de la lesión tumoral.

\section{Caso 3}

Preadolescente de género femenino, de 11 años de edad, hospitalizada para estudio por Infección del Tracto Urinario (ITU) a repetición. Al examen físico abdominal se descubrió masa en hipogastrio.

Se realizaron estudios imagenológicos, donde se apreció tanto en la ecotomografía como en el TAC abdominal (figuras $4 \mathrm{~A}$ y B) una masa con componente sólido quístico vascularizado de 7,8 x 5,5 x 5,7 cm en la pared posterolateral de la vejiga. La lesión infiltraba la pared vesical y comprometía el mesenterio en casi toda su extensión pélvica (figuras $4 \mathrm{C}$ y D). Considerando el riesgo de una cistectomía radical durante el acto quirúrgico, se decidió tomar muestra incisional, cuya histología resultó compatible con TMI vesical. El estudio inmunohistoquímico reveló: Vimentina: positivo; Desmina: positivo; Actina ME: positivo; Citoqueratina: positivo; ALM: positivo y ALK: negativo.

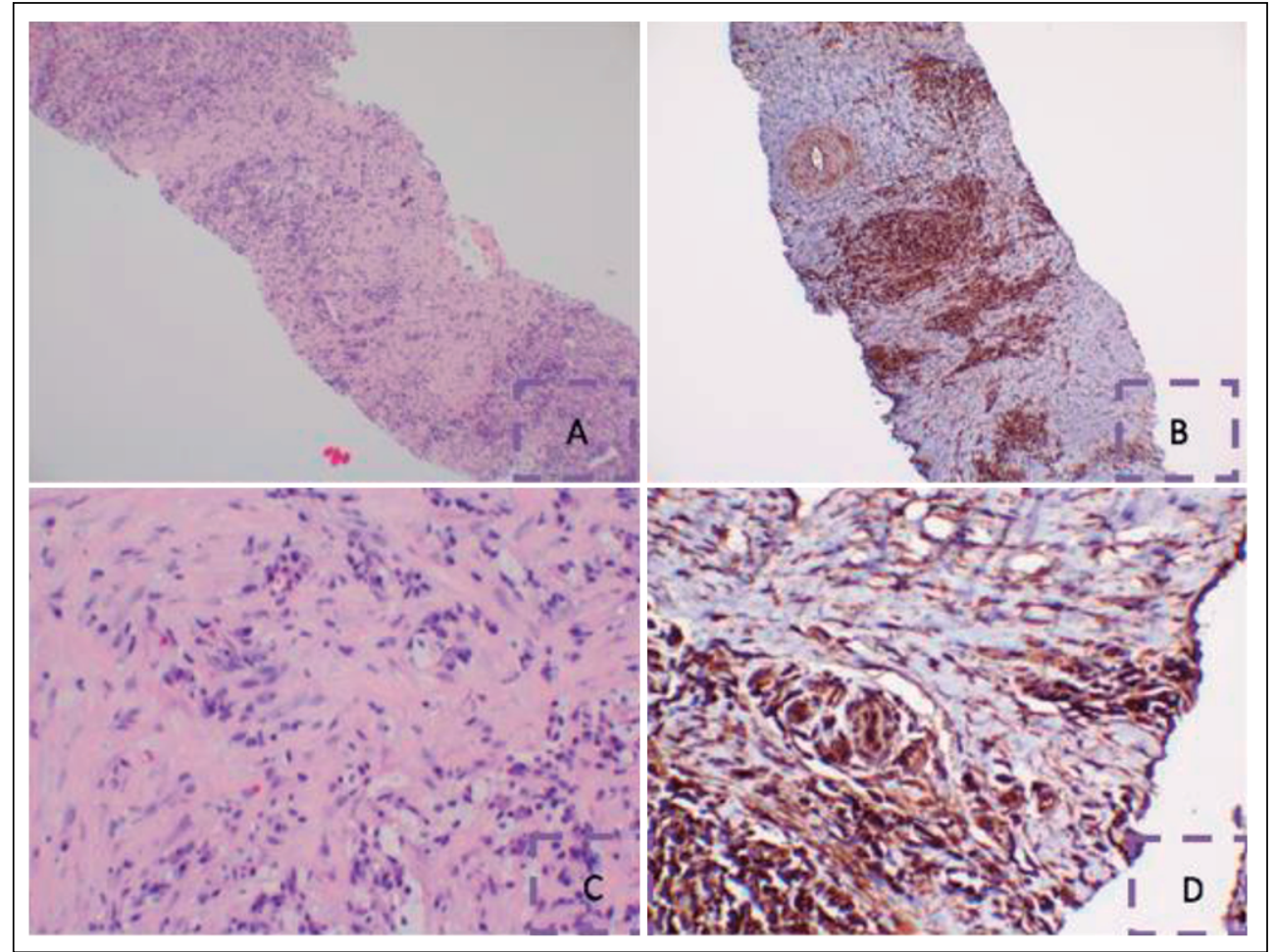

Figura 3. A y C: tinción de HE. B y D: actina ME positivo en células fusadas. 
Se indicó tratamiento con antiinflamatorios de la familia de los COX-2 asociado a Prednisona (no se cuenta con registro de dosis de medicación) por aproximadamente un año, lográndose la remisión del tumor. Tras el período de tratamiento se realizó un control ecográfico pesquisándose una lesión residual limitada a un pequeño segmento de la pared vesical. Mediante cistoscopía que evidenció una lesión mamelonada en la cara posterior de la vejiga (figuras 5Ay B), se delimitó la lesión y se efectuó cistectomía parcial con bordes libres. La biopsia (figuras 5C y D) informó una lesión compatible con TMI asociado a foco de Actinomices, lo que probablemente fue el causante de la reacción inflamatoria que dio origen al tumor. Fue evaluada por infectología, iniciándose terapia antibiótica con Penicilina Sódica EV por 7 días y luego Amoxicilina 1 g cada 12 horas por 6 meses con respuesta favorable.

Actualmente, permanece en controles con oncología, sin evidencia de recidivas.

\section{Discusión}

El TMI, también conocido como pseudotumor inflamatorio $^{1}$, es una neoplasia benigna infrecuente $e^{2,3,12}$, no obstante, está clasificado en el grupo de tumores miofibroblásticos de comportamiento intermedio ${ }^{4,6}$ por su potencial malignidad, caracterizado por invasión a estructuras vecinas, recurrencia y metástasis a distancia, siendo esta última excepcional ${ }^{3,8}$.

A menudo se presenta en niños y adultos jóvenes $^{2,3,5,6}$, con leve preferencia por el género masculino $(\mathrm{H} / \mathrm{M}=1,3 / 1)^{3}$.

$\mathrm{Su}$ etiología es incierta, se sugiere relación con traumatismos, cirugías, patologías autoinmunes, inflamación e infecciones virales o bacterianas como el virus Epstein barr, virus Herpes simplex, Mycobacterias, Mycoplasma, entre otras. Los estudios genéticos comprueban translocación de cromosomas y fusión de genes $^{3,5-8,10}$.

Este tumor se localiza en distintos sitios anatómi$\cos ^{1-3}$, con mayor frecuencia en pulmón ${ }^{7,10}$, sin embargo, recientes trabajos descritos en tabla 1, muestran mayor incidencia en focos extrapulmonares como la cavidad abdominopélvica, de los cuales el hígado es el principal afectado ${ }^{5}$. Sitios atípicos como intestino, páncreas, aparato genitourinario y óseo, también están descritos $^{8,9}$.

La presentación clínica es habitualmente en relación a la localización del tumor, pudiendo ser asin-
Figura 4. A y B: muestran TMl localizado en pared vesical posterior. C y D: se evidencia TMl desplazando estructuras pélvicas hacia la izquierda.

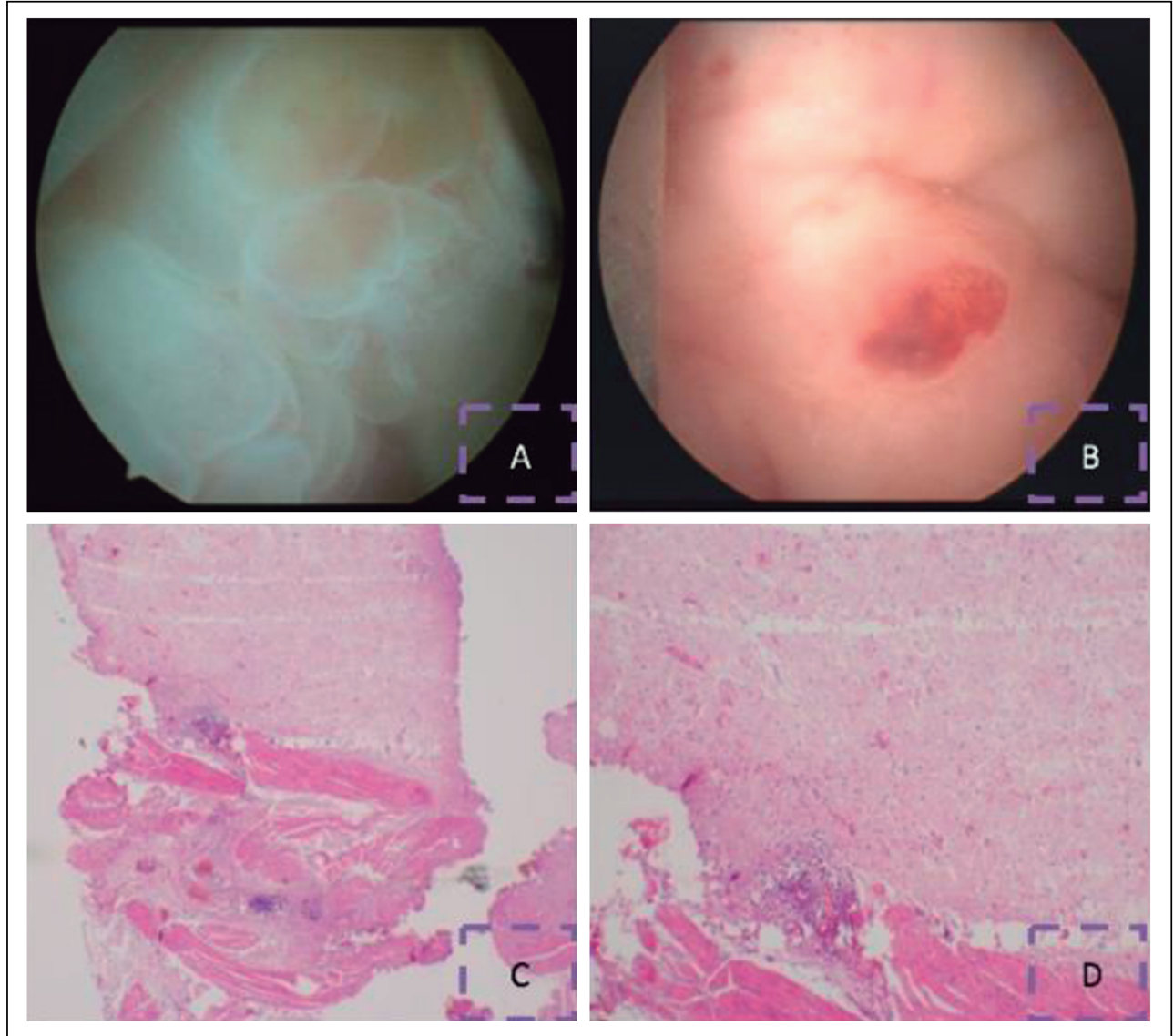



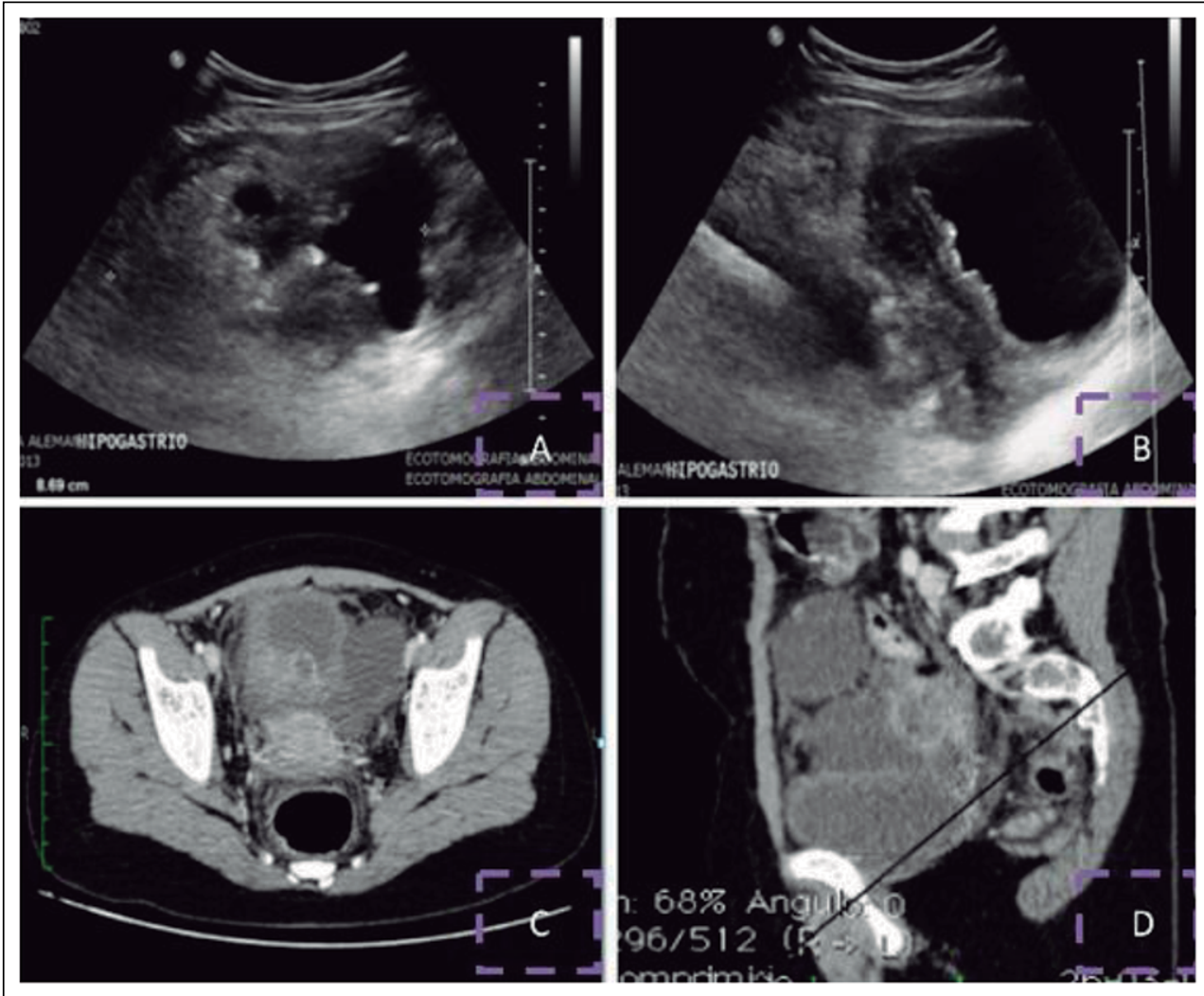

Figura 5. A y B: imagen cistoscópica de TMI. C y D: pared muscular con musculo liso infiltrado por tejido miofibroblástico mixoide con focos de infiltrado linfoplasmocitario y eosinófilos.

tomáticos ${ }^{2,11}$. Aproximadamente el 5 a $10 \%$ tienen manifestaciones sistémicas y constitucionales como fiebre, pérdida de peso, anorexia, anemia microcítica e hipocrómica, hipergamaglobulinemia y trombocitosis. (Probablemente debido a la producción tumoral de IL-1 e IL-6 $)^{4,5}$.

La lesión generalmente es única, aunque pueden ser múltiples en el 5\% de los $\operatorname{casos}^{5}$ y mediante estudios imagenológicos es difícil distinguir de otras neoplasias $^{1,2,5,6}$.

El diagnóstico definitivo se realiza con histología más inmunohistoquímica ${ }^{7,9}$.

Se han descrito 3 patrones histológicos. El vascular mixoide, el compacto de células fusadas con células inflamatorias entremezcladas y el hipocelular fibroso (predominio de matriz colágena densa) ${ }^{3-5,13}$. Wang et $\mathrm{al}^{3}$, evidenciaron que el patrón de células fusadas fue el más frecuente representado en el $96 \%$ de los casos.

El estudio microscópico no es suficiente para diferenciar de otros tumores como: estromales, leiomiosarcomas e histiocitoma fibroso inflamatorio maligno, por lo que es necesaria la inmunohistoquímica para confirmar el diagnóstico. Las células mesenquimales suelen ser inmunorreactivas para vimentina, desmina, SMA y a proteína $\mathrm{S}_{100}{ }^{8}$, y negativos para $\mathrm{CKIT}^{12}$.

Hasta un $71 \%$ de estos tumores son positivos para ALK-1 (anaplastic Lymphoma Kinase) ${ }^{8,12}$, lo que le

\begin{tabular}{lclc}
$\begin{array}{l}\text { Tabla 1. Localización anatómica de tumor miofibroblástico } \\
\text { inflamatorio }\end{array}$ & \\
\hline Autores & $\begin{array}{c}\text { Total de la } \\
\text { muestra }\end{array}$ & Localización & $\begin{array}{c}\% \text { de } \\
\text { pacientes }\end{array}$ \\
\hline Dalto, et al. & 32 & Abdomen y pelvis & $28 \%$ \\
& & Cabeza y cuello & $22 \%$ \\
& & Tórax & $22 \%$ \\
& & Genitourinario & $9 \%$ \\
Karnk, et al. & & Intestino & $6 \%$ \\
& & Hígado & $6 \%$ \\
Wang, et al. & Musculoesquelético & $6 \%$ \\
& & Tórax & $15 \%$ \\
& & Abdominal & $85 \%$ \\
& 23 & Abdomen y pelvis & $74 \%$ \\
& & Pulmón & $8,6 \%$ \\
& & Cabeza y cuello & $4,3 \%$ \\
& & Tronco & $4,3 \%$ \\
& & Extremidades & $8,6 \%$ \\
\hline
\end{tabular}

otorga al TMI mayor susceptibilidad al tratamiento farmacológico que los que no la expresan ${ }^{4}$, pero con un índice más elevado de recurrencia ${ }^{8}$. Los estudios genéticos han determinado que el 50\% de los TMI alojará una alteración de la estructura del gen ALK (cromosoma 2p23) generando varios patrones de fusión ${ }^{7,9,14}$. 
Tateishi Y, et $\mathrm{al}^{9}$, presentan un caso de TMI intraóseo, donde mediante FISH (fluorencence in situ hybridization) se identifica una fusión ATIC-ALK.

El diagnóstico diferencial se hace con lesiones benignas como granuloma de células gigantes, tumor fibroso solitario, mixoepitelioma, mixofibroma ${ }^{7,9}$ y con tumores malignos como sarcoma miofibroblástico de bajo grado, teratomas, rabdomiosarcomas y linfo$\operatorname{mas}^{3,7}$.

El manejo del TMI dependerá de su localización, la expresión de ALK, su comportamiento y la factibilidad de resección quirúrgica.

En relación al comportamiento, se describen casos de infiltración local, recurrencia y metástasis, no obstante, también hay publicaciones que informan la resolución espontánea ${ }^{6,12}$.

Zhao, et $\mathrm{al}^{6}$, presentaron dos casos de pacientes adultos con diagnóstico de tumor miofibroblástico inflamatorio de localización intraabdominal, catalogados como irresecables y que involucionaron sin ningún tratamiento. Además, presentaron una revisión sistemática con un total de 36 pacientes con tumores intraabdominales cuyo comportamiento fue la regresión espontánea sin intervención quirúrgica ${ }^{6}$. La razón de la regresión espontánea no está clara, pero se plantean 3 factores que podrían influir:

1. Localización de la lesión: las lesiones hepáticas tienen mejor pronóstico en contraste con las localizadas en otro sitio del abdomen, pelvis o retroperitoneo.

2. Edad de presentación: la regresión fue más frecuente en edad media y pacientes mayores.

3. Otros factores como aneuploidía, atipias y células similares a células ganglionares, se asociaron a mayor recurrencia y transformación maligna ${ }^{6}$.

En relación al abordaje terapéutico basado en la hipótesis de origen inflamatorio se han reportado casos de uso de antiinflamatorios no esteroidales y corticoides $^{12}$. Se postula que los antiinflamatorios tendrían un efecto inhibitorio sobre la angiogénesis y la proliferación celular a través de la inducción de la apoptosis de fibroblastos y por lo tanto pueden inhibir la vascularización tumoral, la proliferación endotelial y el crecimiento tumoral ${ }^{12}$. Tsuma, et al, presentaron el caso de un adolescente de 13 años de edad con diagnóstico de TMI, manejado con inhibidores de las COX-2 y prednisolona, logrando disminución del tamaño de la lesión para su posterior resección. El análisis inmunohistoquímico reveló la expresión tumoral de COX-2 lo que justificó la eficacia de los inhibidores selectivos de la COX-2 en la reducción del tamaño tumoral ${ }^{14}$.

En la literatura se describen dos casos de pacientes que no respondieron a la terapia con antiinflamatorios y se decidió su manejo con Infliximab, obteniéndose una respuesta favorable y logrando la estabilidad de las lesiones y disminución de la sintomatología en uno de los $\operatorname{casos}^{12}$, y una regresión casi completa de la masa, asociado a ausencia de sintomatología en el otro ${ }^{13}$.

El rol de la radioterapia y quimioterapia aún no está claro ${ }^{4,12}$, ya que, no ha mostrado un beneficio definitivo $^{5}$, siendo el tratamiento de elección la resección quirúrgica la cual es curativa ${ }^{1,3,5,8,12}$.

La recurrencia va del 25 al 40\%, y es más frecuente en lesiones extrapulmonares y durante el primer año tras la resección ${ }^{3,5,7}$. Si la extirpación es completa y la lesión muestra márgenes libres, el índice de recurrencia es menor al $10 \%{ }^{3}$. Las metástasis son infrecuentes y en alrededor de un $2 \%{ }^{8,11}$.

Las características que pueden predecir un peor pronóstico, recurrencia y probabilidad de metástasis son: género femenino, edad sobre los 25 años, ubicación abdominopélvica, gran tamaño, masa multinodular, resección incompleta y ALK negativo ${ }^{12}$.

Dalton B. et al., evaluaron 32 pacientes con diagnóstico de TMI, logrando determinar una mayor asociación a mortalidad la persistencia y la recurrencia de la enfermedad ( 67 vs $0 \%)^{1}$.

En cuanto al seguimiento se sugiere tras la resección un seguimiento ecográfico, a los 3, 6 y 12 meses $^{1,3}$.

\section{Conclusión}

El TMI está catalogado como un tumor miofibroblástico de comportamiento intermedio. Sus manifestaciones clínicas son variadas y estarán determinadas por el sitio anatómico afectado. El manejo dependerá de su localización, expresión de ALK, comportamiento y la factibilidad de resección quirúrgica.

En este trabajo se presentaron 3 casos con manifestaciones clínicas, manejos y resultados distintos, lo que apoya la diversidad de esta entidad. La utilización de anticuerpos monoclonales está descrito, hasta el momento de la revisión, en sólo 3 pacientes a nivel mundial y nuestro caso es el segundo descrito en Chile, si bien sus resultados parecen ser alentadores, se requieren más estudios para establecer como esquema de terapia en el manejo de los TMI ALK negativos no susceptibles a resección quirúrgica.

\section{Responsabilidades Éticas}

Protección de personas y animales: Los autores declaran que los procedimientos seguidos se conformaron a las normas éticas del comité de experimentación humana responsable y de acuerdo con la Asociación Médica Mundial y la Declaración de Helsinki. 
Confidencialidad de los datos: Los autores declaran que han seguido los protocolos de su centro de trabajo sobre la publicación de datos de pacientes.

Derecho a la privacidad y consentimiento informado: Los autores han obtenido el consentimiento informado de los pacientes y/o sujetos referidos en el artículo. Este documento obra en poder del autor de correspondencia.

\section{Conflicto de intereses}

Los autores declaran no tener conflicto de intereses.

\section{Referencias}

1. Dalton B, Thomas P, Sharp N, et al. Inflammatory myofibroblastic tumors in children. J Pediatr Surg. 2016;51:541-4.

2. Karnak I, Senocak M, Ciftci A, et al. Inflammatory Myofibroblastic Tumor in Children: Diagnosis and Treatment. J Pediatr Surg. 2001;36(6):908-12.

3. Wang Z, Zhao X, Li K, et al. Analysis of clinical features and outcomes for inflammatory myofibroblastic tumors in China: 11 years of experience at a single center. Pediatr Surg Int 2016;32:239-43.

4. Pfeifer JD, Dehner LP. Soft tissue and Bone. En: Humphrey ed. The Washington manual Of Surgical Pathology, segunda edición, Washington DC. Wolters Kluwer Health/Lippincott Williams y Wilkins. 2012. pp 754-5.

5. Osnaya $\mathrm{H}$, Zaragoza $\mathrm{T}$, Escoto J, et al. Tumor miofibroblástico inflamatorio (pseudotumor inflamatorio) ocasionando abdomen agudo. Rev Chil Cir. 2014;66(3):264-8.
6. Zhao JJ, Ling JQ, Fang Y, et al. Intraabdominal inflammatory myofibroblastic tumor: Spontaneous regression. World J Gastroenterol. 2014;20(37):13625-31.

7. Liu HK, Lin YC, Yeh ML, Chen YS, Su YT, Tsai CC. Inflammatory myofibroblastic tumors of the pancreas in children A case report and literature review. Medicine 2017;96:2(e5870).

8. Oeconomopoulou A, de Verney Y, Kanavaki K, Stefanaki K, Pavlakis K, Salakos C. Inflammatory myofibroblastic tumor of the small intestine mimicking acute appendicitis: a case report and review of the literature. J Med Case Rep. 2016;10:100.

9. Tateishi Y, Okudela K, Kawai S, et al. Intraosseous inflammatory myofibroblastic tumor of the mandible with a novel ATIC-ALK fusion mutation: a case report. Diagn Pathol. 2016;11:132.

10. Izumi G, Narugami M, Saita $Y$, et al. Successful sleeve lobectomy of pediatric inflammatory myofibroblastic tumor. Pediatr Int. 2016;58:1087-9.
11. Delgado Duatis G, Bejar Sánchez R, Alonso Jiménez L. Invaginación atípica por tumor miofibroblástico inflamatorio. Anales de pediatría. 2015;83(3):221-2.

12. Grunoholz D, Appiani F, Abarca C, Manriquez M, Pinilla J, Wainstein E. Tumor miofibroblástico peritoneal. Respuesta favorable asociada a infliximab. Caso clínico. Rev Med Chile 2015;143:943-7.

13. Germandis G, Xanthakis I, Tsitouridis I, et al. Regression of Inflammatory Miofibroblastic Tumor of the Gastrointestinal Tract Under Infliximab Treatment. Digestive diseases and Sciences. 2005;50(2):262-5. DOI: $10.1007 / \mathrm{s}$ 10620-005-1593-1.

14. Tsuma Y, Miyachi M, Ouchi K, et al. Neoadyuvante Treatment With Cyclooxygenase-2 inhibitor and Prednisolona Allows Conservative Sugery for Inflamatory Myofibroblastic Tumor of the Bladder. Journal Pediatrics Hematology Oncology 2016;38:e283-5. 